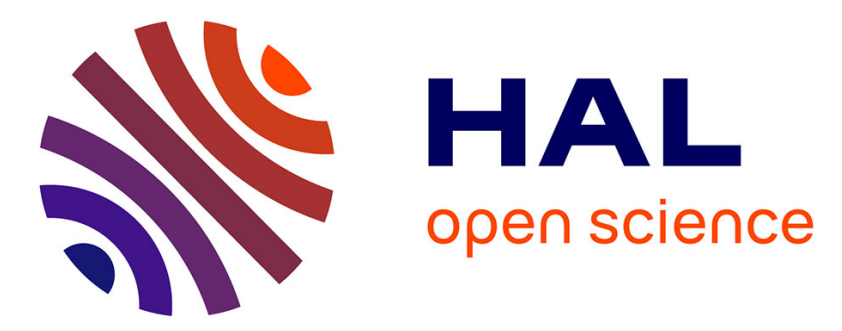

\title{
Immigration, langue, école et citoyenneté au Japon : l'école japonaise face à la scolarisation des enfants d'immigrants
}

Christian Galan

\section{- To cite this version:}

Christian Galan. Immigration, langue, école et citoyenneté au Japon: l'école japonaise face à la scolarisation des enfants d'immigrants. Revue Française de Pédagogie, 2005, 153 (1), pp.109-119. 10.3406/rfp.2005.3398 . hal-02399591

\section{HAL Id: hal-02399591 https://hal.science/hal-02399591}

Submitted on 9 Dec 2019

HAL is a multi-disciplinary open access archive for the deposit and dissemination of scientific research documents, whether they are published or not. The documents may come from teaching and research institutions in France or abroad, or from public or private research centers.
L'archive ouverte pluridisciplinaire HAL, est destinée au dépôt et à la diffusion de documents scientifiques de niveau recherche, publiés ou non, émanant des établissements d'enseignement et de recherche français ou étrangers, des laboratoires publics ou privés. 


\section{Immigration, langue, école et citoyenneté au Japon : l'école} japonaise face à la scolarisation des enfants d'immigrants Mr Christian Galan

\section{Citer ce document / Cite this document :}

Galan Christian. Immigration, langue, école et citoyenneté au Japon : l'école japonaise face à la scolarisation des enfants d'immigrants. In: Revue française de pédagogie, volume 153, 2005. pp. 109-119;

doi : https://doi.org/10.3406/rfp.2005.3398

https://www.persee.fr/doc/rfp_0556-7807_2005_num_153_1_3398

Fichier pdf généré le 22/04/2018 


\title{
Resumen
}

Christian Galan -Inmigración, idioma y ciudadanía en Japón: la escuela japonesa frente a la escolarización de los niños de inmigrantes. Japón conoce una crisis demográfica nunca vista que, en los próximos años, va a traer a este país un número de inmigrantes cada vez más elevado. Entonces se plantearán - ya se plantean - la cuestión de la integración de estos inmigrados y de sus hijos y, por lo tanto, la de una nueva definición de la ciudadanía japonesa y de la «japoneidad». Estas cuestiones se discutirán en este artículo después de que hayamos presentado los diferentes obstáculos relacionados con la lengua escrita y con el sistema educativo japonés que se oponen hoy en día a la integración de estos extranjeros. Después de describir la situación actual de los niños extranjeros en Japón y la manera en que se administra su presencia en las escuelas del archipiélago, discutiremos igualmente la capacidad de la escuela japonesa de asegurar la misión, nueva para ella, que constituye el hecho de conducir poblaciones al principio no japonófonas al dominio de la lengua japonesa oral y escrita, y de las consecuencias que esta nueva situación podría tener sobre el conjunto del sistema educativo y de las prácticas pedagógicas de este país.

\section{Résumé}

Le Japon connaît une crise démographique sans précédent qui, dans les prochaines années, va amener dans ce pays un nombre de plus en plus grand d'immigrants. Se poseront alors - se posent déjà - la question de l'intégration de ces immigrés et de leurs enfants et, partant, celle d'une redéfinition de la citoyenneté japonaise et de la «japonité». Ces questions seront discutées dans cet article après que nous ayons présenté les différents obstacles liés à la langue écrite et au système éducatif japonais qui s'opposent aujourd'hui à l'intégration de ces étrangers. Après avoir décrit la situation actuelle des enfants étrangers au Japon et la façon dont est gérée leur présence dans les écoles de l'archipel, nous discuterons également de la capacité de l'école japonaise à assurer la mission, nouvelle pour elle, que constitue le fait d'amener des populations à l'origine non japonophones à la maîtrise de la langue japonaise orale et écrite, et des conséquences que cette situation nouvelle pourrait avoir sur l'ensemble du système éducatif et des pratiques pédagogiques de ce pays.

\begin{abstract}
Christian Galan -Immigration, language, school and citizenship in Japan: Japanese schools faced with schooling the children of immigrants. Japan is going through an unprecedented demographic crisis which will bring into this country a greater and greater number of immigrants in the years to come. Therefore the question of integrating those immigrants and their children will be is already raised and thus the question of re-defining Japanese citizenship and "Japaneseness" is as well. Those questions will be discussed in this article after we have presented the various obstacles related to the written language and the Japanese education system which currently work against integrating those foreigners. After describing foreign children's current situation in Japan and the way their presence in the country's schools is being handled, we will also discuss the ability of the Japanese schools to fulfil their task - new to them - of leading a non-Japanese speaking population towards mastering the oral and written Japanese language. We will also discuss the consequences that this new situation could have on the whole Japanese education system and its teaching strategies.
\end{abstract}

\section{Zusammenfassung}

Christian Galan -Einwanderung, Sprache, Schule und Bürgerschaft in Japan: die japanische Schule vor dem Schulbesuch eingewanderter Kinder. Japan kennt eine einmalige demographische Krise, die in den nächsten Jahren immer mehr Zuwanderer ins Land führen wird. Dann stellt sich das Problem der Integration dieser Einwanderer und ihrer Kinder, und von daher das Problem einer Neudefinierung der japanischen Bürgerschaft und des "Japantums". Im vorliegenden Artikel werden diese Fragen erörtert, nachdem wir die verschiedenen Hindernisse vorgestellt haben, die mit der schriftlichen Sprache und dem Schulsystem verbunden sind und 
Schulsystem und über die pädagogischen Praktiken haben könnte.

* Schule, die von vielen Kindern aus problembelasteten Familien besucht wird. (lernbehinderte bzw. verhaltensauffällige Schüler) 


\section{Varia}

\section{Immigration, langue, école et citoyenneté au Japon : l'école japonaise face à la scolarisation des enfants d'immigrants}

Christian Galan

Le Japon connaît une crise démographique sans précédent qui, dans les prochaines années, va amener dans ce pays un nombre de plus en plus grand d'immigrants. Se poseront alors - se posent déjà - la question de l'intégration de ces immigrés et de leurs enfants et, partant, celle d'une redéfinition de la citoyenneté japonaise et de la "japonité ». Ces questions seront discutées dans cet article après que nous ayons présenté les différents obstacles liés à la langue écrite et au système éducatif japonais qui s'opposent aujourd'hui à l'intégration de ces étrangers. Après avoir décrit la situation actuelle des enfants étrangers au Japon et la façon dont est gérée leur présence dans les écoles de l'archipel, nous discuterons également de la capacité de l'école japonaise à assurer la mission, nouvelle pour elle, que constitue le fait d'amener des populations à l'origine non japonophones à la maîtrise de la langue japonaise orale et écrite, et des conséquences que cette situation nouvelle pourrait avoir sur l'ensemble du système éducatif et des pratiques pédagogiques de ce pays.

Descripteurs (TEE) : apprentissage des langues, immigration, Japon, lecture, scolarisation, système d'enseignement.

a situation linguistique du Japon est en train Ld'évoluer petit à petit sous l'action conjuguée d'une dramatique baisse démographique et d'un processus de globalisation ou de mondialisation qui conditionnent aujourd'hui autant les orientations politiques de ce pays que son économie.

Toutefois, s'il est évident que la population japonaise s'ouvre toujours plus aux autres cultures et aux autres langues, et si toujours plus de langues étrangères se parlent dans Tôkyô et dans les autres grandes villes du Japon, la situation linguistique réelle des étrangers résidents ne se laisse pas saisir facilement. Ces locuteurs partagent-ils avec leurs hôtes japonais la maîtrise de la langue japonaise? Cette maîtrise englobe-t-elle vraiment celle de l'écrit ? Qu'implique cette maîtrise sur le plan de l'apprentissage? Les " nouveaux arrivants » ont-ils les moyens de mener à bien cet apprentissage au Japon même ou bien avant leur arrivée dans l'archipel ? Qu'en est-il de leurs enfants si ceux-ci séjournent au Japon avec eux, etc. ? Toutes ces questions, qui ont servi de point de départ à notre réflexion, conduisent bien sûr à s'interroger in fine sur les capacités du système éducatif japonais à assurer la mission, nouvelle pour lui, que constitue le 
fait d'amener des populations à l'origine non japonophones à la maîtrise de la langue japonaise orale et écrite.

Aborder ces questions à partir des situations (des expériences) que connaissent les autres grands pays industriels - et notamment la France - amène à raisonner à partir du concept de "barrière ", et à chercher ainsi tout d'abord les obstacles, propres à la situation japonaise, qui se dressent sur la route des populations immigrées ou étrangères désireuses de parvenir à la maîtrise de la langue de leur pays d'accueil. C'est donc ce que nous ferons dans un premier temps avant de nous intéresser ensuite à la situation actuelle de la scolarisation des enfants étrangers.

\section{SPÉCIFICITÉ DE LA LANGUE ÉCRITE JAPONAISE ET STRATÉGIE D'ENSEIGNEMENT}

La première barrière qui fait obstacle à la construction d'un véritable bilinguisme incluant une maîtrise généralisée et partagée de la langue écrite japonaise est, à l'évidence, cette langue écrite elle-même, ou, dit autrement, le fait que la spécificité de la langue écrite japonaise induise des contraintes particulières sur le plan de l'apprentissage - notamment son étalement sur un grand nombre d'années -, contraintes qui ont conduit les pédagogues japonais à mettre en place une méthode d'enseignement de la lecture et de l'écriture elle-même extrêmement contraignante et très précisément programmée dans le temps (1).

La langue écrite japonaise mêle en effet deux grands types de signes: les kana, une cinquantaine de signes syllabiques à la correspondance phoniegraphie quasiment parfaite (un signe = un son), et les kanji, dénomination japonaise des caractères chinois tels que les habitants de l'archipel se les sont progressivement appropriés au fil des siècles. Le nombre des kanji est de plusieurs milliers et ceux-ci présentent par ailleurs la particularité de posséder chacun différentes lectures (le plus souvent deux) en fonction des mots et des contextes dans lesquels ils apparaissent. La stratégie d'apprentissage des kanji retenue à l'école élémentaire depuis la fin du $\mathrm{XIX}^{\mathrm{e}}$ siècle, tenant compte à la fois de cette réalité scripturale unique et de la nécessité de contourner l'obstacle majeur que constitue le long apprentissage des kanji, se résume en trois mots : "mémorisation ", «accumulation » et "substitution ». Mémorisation initiale des kana, puis mémorisation et accumulation d'un premier millier de kanji (lecture(s) et écriture) étalée sur les six années de la scolarité élémentaire (avant d'en étudier mille de plus durant les trois années de collège) et substitution progressive des kanji étudiés aux kana, dans les phrases proposées aux enfants ou dans celles que ceux-ci écrivent.

Cette méthode, du fait de son caractère cumulatif et de l'étalement dans le temps qu'elle implique présente, pour le sujet qui nous intéresse ici, la particularité majeure d'interdire à quiconque de commencer son apprentissage à un niveau autre que le niveau zéro, théoriquement celui de la première année de l'école élémentaire, sauf à pouvoir rattraper son retard d'une façon ou d'une autre. Mais, à l'exception des individus doués d'une mémoire prodigieuse ou dotés de capacités de travail quasi surhumaines, le facteur temps et les limites de la mémoire humaine ordinaire constituent - à ce jour - deux des principales barrières qui se dressent sur la route ardue qui mène à la maîtrise de l'écrit japonais.

\section{CHOIX ORGANISATIONNELS ET CONCEPTION DE L'ENFANT}

Un second ensemble d'obstacles ou de barrières nous paraît être constitué par différents choix opérés dans la structure et l'organisation du système éducatif ainsi que par certains usages pédagogiques, si ce n'est par la conception même de l'enfant qui prédomine dans la société japonaise.

\section{L'éducation préélémentaire}

Le système éducatif japonais n'intégrant pas véritablement le niveau préélémentaire (2), les directives officielles du ministère de l'Éducation fixent le début de l'apprentissage des kana, et donc de la lecture, au moment de l'entrée à l'école élémentaire, shôgakkô. Les directives concernant les écoles préélémentaires précisent de leur côté que seul un travail de sensibilisation à la langue doit être entrepris dans ces écoles.

"Officiellement » donc, les enfants n'apprennent pas les kana avant leur entrée à l'école élémentaire. La réalité cependant est bien différente. En effet, si tous les instituteurs enseignant en première année de l'école élémentaire font comme si les enfants ne connaissaient aucun kana au premier jour de l'école, dans la réalité, c'est loin d'être le cas. Plus de $90 \%$ des nouveaux élèves les connaissent en effet déjà tous parfaitement, les autres les ayant mémorisés en partie. C'est qu'ils les ont appris avec leurs parents, 
leur mère le plus souvent, la spécificité des kana, un signe $=$ un son, facilitant grandement leur apprentissage : il ne viendrait à l'idée d'aucun parent japonais qu'il pourrait échouer à faire apprendre ces signes à leur(s) enfant(s), ni d'ailleurs qu'il s'agit là pour ce(s) dernier(s) de quelque chose de difficile.

Cette situation a une double conséquence pour les enfants étrangers dont le japonais n'est pas la langue d'au moins un des parents:

- à supposer que leurs parents aient les moyens de leur donner une scolarité préélémentaire - payante, car non obligatoire -, celle-ci leur permettra dans la majorité des cas de se socialiser, de s'épanouir et développer leur maîtrise du japonais parlé, mais ne les initiera pas, ou pas suffisamment, à l'écrit ;

- au moment de l'entrée à l'école élémentaire, ces enfants auront peut-être le niveau linguistique souhaité par les directives officielles, mais, en réalité, sur le plan de la maîtrise de l'écrit, ils seront « en retard " par rapport aux enfants japonais. Alors que ces derniers ne feront que réviser des choses déjà apprises et familières, ils seront, eux, en situation de vrai apprentissage : l'institution scolaire telle qu'elle est organisée aujourd'hui ne leur donnera donc pas la possibilité de compenser, avant le début de la scolarité obligatoire, le handicap que constitue sur ce plan le fait de grandir dans un foyer dans lequel l'écrit japonais n'est pas utilisé, voire simplement connu.

\section{L'école, lieu de validation de l'apprentissage de la lecture}

Or, non seulement ces enfants étrangers vont arriver à l'école élémentaire avec des niveaux d'acquisition et de pratique de la langue écrite différents de ceux de l'immense majorité des enfants japonais, mais, en plus, l'école élémentaire elle-même ne leur permettra pas de rattraper ce retard - sauf si des cours complémentaires sont mis spécialement mis en place pour eux.

En effet, comme nous l'avons montré dans nos travaux antérieurs (Galan, 2001) les leçons de "langue japonaise", kokugo, du premier trimestre de la première année de l'école élémentaire sont davantage des leçons de contrôle ou de révision que des leçons d'apprentissage proprement dit (ce qui peut se comprendre étant donné, on vient de le voir, que la quasi-totalité des enfants japonais connaissent déjà les kana).
Dans le même ordre d'idées, et cela est également d'une grande importance pour le sujet qui nous intéresse ici, pour une grande partie du travail d'apprentissage des kanji - et donc de la lecture - à partir de la quatrième année de l'école élémentaire sinon dès la fin de la troisième année dans certaines écoles -, les instituteurs s'en remettent au travail personnel des enfants: le rôle des parents et, souvent, des juku (3), ces "écoles d'après l'école » que fréquentent de nombreux petits Japonais, devenant par là même extrêmement important à ce niveau du processus d'apprentissage.

Qu'en est-il ou qu'en sera-t-il de la viabilité de cette méthode dans le cas d'enfants étrangers dont les parents ne maîtrisent pas l'écrit japonais ou ne peuvent pas forcément scolariser ceux-ci deux fois, une fois à l'école publique et une fois en juku?

\section{La double finalité des leçons de lecture}

Une autre barrière nous paraît être constituée par le fait que, d'une façon générale, l'école élémentaire au Japon est autant, sinon plus, un lieu de socialisation des enfants - et d'apprentissage de la " japonité " - qu'un lieu d'apprentissage des savoirs académiques. Cela se vérifie dans le fonctionnement général de l'école, dans l'ensemble des disciplines, et tout particulièrement dans l'enseignement de la langue et de la lecture.

Ainsi, pour ne prendre qu'un seul exemple, parmi les directives officielles de l'école élémentaire publiées en 1989 et entrées en application à partir de 1992, une nouvelle directive fixe les dix critères selon lesquels doivent être choisis les textes proposés aux enfants. Or, plus de la moitié de ces critères toujours en vigueur relèvent de préoccupations n'ayant rien à voir avec la lecture et reflètent uniquement la volonté du ministère de renforcer l'enseignement de la morale. Plus que jamais, les directives de 1989, comme les suivantes de 1999, vont dans le sens de ce que Sakamoto Takahiko et Makita Kiyoshi écrivaient en 1973 à propos de ce qui constitue la ligne officielle de l'enseignement de la lecture au Japon depuis 1958: "L'objectif principal de l'enseignement de la lecture au Japon n'est pas seulement que les enfants sachent lire mais également qu'ils puissent comprendre la morale des histoires et qu'ils développent des personnalités saines. " (Makita \& Sakamoto, 1973, p. 450)

II n'est pas sûr cependant que des mesures de ce genre, et à travers elles la pédagogie qui est aujour- 
d'hui appliquée dans les écoles, facilitent l'apprentissage de la lecture par les enfants étrangers: les directives qui favorisent la culture japonaise et les valeurs propres à la «japonité » ne constituent-elles pas un véritable handicap pour ceux qui ne partagent pas ces valeurs du simple fait que leurs parents, élevés dans d'autres cultures, ne les connaissent pas eux-mêmes et ne peuvent donc pas les transmettre?

\section{La conception de l'enfant}

Le dernier point qui nous paraît poser problème est lié à la conception même de l'enfant qui prévaut dans l'ensemble de la société japonaise et qui s'articule autour de trois grands principes de base: 1) tous les enfants ont un potentiel intellectuel égal sinon équivalent ; 2 ) tous les enfants ont la capacité de bien étudier et d'assimiler les programmes; 3) des habitudes telles que l'application et l'attention qui sont à la base de toute réussite scolaire peuvent et doivent être enseignées.

L'acceptation de ces principes par tous, parents et enseignants, fait que les différences qui apparaissent entre les enfants sont considérées comme résultant de la qualité du travail et des efforts fournis par chacun d'eux, ainsi que de leur persévérance, et non de leur seul potentiel individuel. Ces trois principes excluent également de fait toute référence au milieu social ou intellectuel dans lequel vit l'enfant, comme si l'un et l'autre milieux étaient non agissants, n'avaient pas d'influence sur les performances des enfants.

Cette conception des capacités de l'enfant aboutit également au refus de remettre en cause la pédagogie utilisée, mais plus encore, pour le sujet qui nous intéresse ici, au refus de tout échec scolaire. En effet, $\mathrm{si}$, au Japon, le passage dans la classe supérieure est automatique (sauf cas exceptionnel de très longue absence due à la maladie), c'est sans doute en premier lieu parce que faire redoubler un enfant est considéré comme néfaste, puisque cela l'exclut du groupe auquel il appartient (l'effet psychologique produit serait pire que le retard accumulé) ; mais c'est aussi parce que, selon les principes que nous venons d'énoncer, les Japonais considèrent que les retards ou les difficultés peuvent être compensés par un surplus de travail et d'efforts. Inutile donc de faire perdre une année à celui qui présente quelque retard: il pourra le combler par un enseignement spécial, ou plutôt par un temps de travail plus long, aménagé le plus souvent en dehors des heures de cours.
L'apprentissage de la lecture ne fait pas exception à la règle. Fondée sur le présupposé que tous les enfants peuvent le maîtriser, qu'ils ont tous au départ les mêmes capacités pour y arriver, quels que soient leurs antécédents, la méthode retenue à l'école tient donc très peu compte des particularités de chaque enfant et met surtout l'accent sur l'acquisition des seuls mécanismes de lecture. II s'agit là encore d'un élément important lorsqu'on s'intéresse aux enfants étrangers scolarisés au Japon, car ce présupposé transforme en obstacle toute différence, à commencer par celle qui, par définition, caractérise ces enfants.

\section{Un enseignement scolaire non adapté à la différence}

En résumé, au travers de tous les points concrets que nous venons d'évoquer et qui concernent l'apprentissage de la langue japonaise, il apparaît que l'école, telle qu'elle fonctionne aujourd'hui au Japon sur le plan organisationnel aussi bien que pédagogique, ne semble pas prête à (bien) accueillir les enfants étrangers. Actuellement, en effet, l'école japonaise "apprend à lire » à des enfants japonais, nés de parents japonais, élevés au Japon, parlant le japonais depuis leur naissance et vivant dans un entourage ou la seule langue parlée, entendue, écrite et lue est le japonais - et, sauf cas anecdotique, les enseignants japonais dans leur immense majorité, ne connaissant que cette situation, ne sont pas préparés à faire face à un autre public.

Cela n'a, en un sens rien de surprenant, puisque l'école japonaise depuis les années 1950 a été pensée et voulue pour donner à tous les enfants du nord au sud du Japon un enseignement identique en tous points : rythme, progression, matériaux utilisés, etc. Organisée sur la négation des différences qui peuvent exister entre les enfants, elle n'a pas été prévue, bien sûr, sauf à faire appel à la bonne volonté individuelle de certains enseignants, pour accepter et éduquer avec efficacité des enfants par définition "très différents". Et encore ne parlonsnous pas du cas de ces enfants qui pourraient arriver à des âges nécessitant par exemple une scolarisation en collège...

Comment dans ces conditions l'école japonaise pourrait-elle gérer le cas des enfants étrangers dans un système qui par ailleurs, on l'a vu, refuse le concept même d'échec scolaire? En l'état actuel cela nous paraît quasiment impossible, à moins, comme on va le voir à présent, d'évacuer ce problème de l'école... 


\section{DÉMOGRAPHIE, IMMIGRATION ET ÉCOLES}

Sur la base des chiffres actuels de la natalité, les projections du Bureau de la statistique du Japon évaluent que dans un siècle (2100) la population de l'archipel sera réduite à presque la moitié $(67 \mathrm{mil}-$ lions) de celle d'aujourd'hui (127 millions). Quelle que soit la solution globale que les autorités japonaises choisiront pour faire face à la pénurie de main-d'œuvre qui s'annonce - on prévoit, en 2050, un retraité pour un actif et demi (Sômushô, 2000), il est désormais difficile de penser qu'elles puissent éviter d'avoir recours à une immigration étrangère importante. II n'apparaît pas moins évident cependant que cette politique sera fortement contrôlée et encadrée, même si le sujet très polémique reste peu débattu dans les médias et curieusement peu présent dans les rapports officiels.

La politique d'immigration définie par les derniers gouvernements japonais (voir par exemple Shushô Kantei, 2000) se laisse entrevoir largement dans les efforts développés au Japon et dans le reste du monde afin de promouvoir l'étude et la diffusion de la langue et de la civilisation japonaises. Pendant longtemps, les étrangers séjournant au Japon pouvaient en effet se caractériser sinon par leur capacité à parler, lire et écrire la langue japonaise, du moins par leur volonté propre d'acquérir cette capacité. Jusqu'à présent, des étrangers pouvaient certes passer plusieurs années au Japon sans jamais accéder à la maîtrise de la lecture en japonais, ou, plus rarement, sans même parler la langue, mais ces étrangers étaient loin d'être majoritaires, y compris au sein des immigrés "historiques", les Coréens notamment, qui avaient une excellente maîtrise du japonais. Des couples mixtes se constituaient et s'établissaient dans l'archipel, mais il y avait relativement peu de couples non mixtes établis au Japon pour une longue durée. II y avait d'une façon générale peu d'étrangers au Japon et par voie de conséquence très très peu d'enfants étrangers dans les écoles japonaises. Ce qui nous faisait écrire, il y a maintenant plus de quinze ans, quand nous avons commencé à nous intéresser à l'enseignement de la lecture au Japon, que les instituteurs de ce pays enseignaient dans un contexte absolument inouï pour leurs alter ego européens - et en tout cas français : ils avaient en effet, à n'importe quel niveau qu'ils enseignassent, non seulement la certitude d'avoir en face d'eux des enfants qui maîtrisaient tous la langue japonaise (4), mais également celle que les parents de ces enfants, dans leur immense majorité, parlaient et comprenaient eux aussi cette langue, ayant été eux-mêmes, dans leur quasi totalité, scolarisés dans le système éducatif japonais.

C'est cette situation qui est cependant aujourd'hui appelée à évoluer. Une immigration non japonophone en grande partie issue de pays culturellement non sinisés, et essentiellement économique, est en train de prendre le pas sur toutes les formes d'immigration que le Japon avait connues jusqu'ici.

Le nombre des étrangers légalement enregistrés au Japon est passé de 782910 en 1980 (soit 0,67\% d'une population totale estimée à 117060000$)$ à 1915030 en 2003 (soit 1,5\% d'une population totale estimée à 127619000 ), et plus de $65 \%$ sont des primo-arrivants. Les Asiatiques qui représentaient $93,81 \%$ de l'ensemble des immigrants en 1980 (734 476) n'en représentaient plus que 74,3\% en 2003 (1 422 979). Par ailleurs, si le nombre des Coréens est resté stable sur cette période, oscillant entre 680000 et 613000 , celui des Chinois, en revanche, est passé de 52896 en 1980 à 462396 en 2002 (près de neuf fois plus), et il en est allé de même de toutes les autres nationalités asiatiques - les Philippins notamment ont vu leur nombre passer de 5547 en 1980 à 185237 en 2003 (plus de trente trois fois plus). L'autre élément marquant de l'immigration au Japon, suite à la politique d'ouverture ciblée du gouvernement japonais dans les années 1990, est, même si celle-ci semble aujourd'hui marquer le pas, l'augmentation considérable du nombre des immigrés en provenance d'Amérique du Sud, un nombre qui est passé de 2719 en 1980 à 343635 en 2003 (plus de cent vingt six fois plus), et notamment en provenance du Brésil dont le nombre d'immigrants est passé durant la même période de 1492 à 274700 (près de cent quatre-vingt cinq fois plus !). II s'agissait d'ailleurs essentiellement, dans le cas de ces derniers, de nikkeijin, c'est-à-dire de descendants de migrants d'origine japonaise dont les familles s'étaient installées en Amérique du Sud à partir de la fin du XIXe siècle. Quant à l'immigration en provenance de l'Europe, de la Russie, des ÉtatsUnis, du Canada, de l'Australie et de la Nouvelle Zélande, elle est passé de 41499 immigrants en 1980 à 136510 en 2004 (Hômushô, 2003).

Un rapport de I'OCDE paru en janvier 2004 met en avant le phénomène mondial que constituent les "nouveaux travailleurs immigrés ", c'est-à-dire des travailleurs dont la motivation est la recherche d'un emploi vers (ou entre) les pays les plus prospères de la planète. Vieillissement démographique et pénurie 
de main-d'œuvre sont les deux principales raisons pour lesquelles les pays d'accueil encouragent ce mouvement : le Japon se retrouve tout à fait dans ce cas de figure, sauf qu'il n'a pas encore choisi d'ouvrir ses frontières et qu'il se demande comment le faire. Ces "nouveaux travailleurs immigrés " comptent en leur sein aussi bien des ouvriers qualifiés que des migrants moins bien ou pas du tout formés. Leur installation dans le pays d'accueil est soit définitive soit temporaire, en fonction de la politique en vigueur, mais, même dans ce dernier cas, il s'agit le plus souvent d'un "temporaire" qui, du côté des immigrants, cherche à s'inscrire dans la durée.

Un document de synthèse publié en France et relatif aux différents projets des immigrants distingue au sein de ces derniers (Enel \& Delesalle, 2001, p. 1) :

«- les cosmopolites : cadres de haut niveau ou, à l'inverse, personnes faiblement qualifiées qui se déplacent de pays en pays au gré des opportunités d'emploi ;

" - les primo-arrivants, dont l'installation durable [...] est conditionnée à leur intégration positive dans le pays ;

«- les primo-arrivants, qui ont pour projet explicite de [prendre la nationalité du pays d'accueil] ;

«- les primo-arrivants sans projet, soit parce qu'ils n'ont pas eu le temps de l'élaborer, soit parce qu'ils vivent à l'ombre du conjoint ou de la famille ".

Ce sont à l'évidence les trois dernières catégories qui vont constituer un véritable défi pour le Japon dans les années futures. Or, le seul objectif de ces nouveaux immigrants étant économique, leur investissement dans l'étude de la langue japonaise - si leur travail leur en laisse le temps - sera minime et ne leur permettra vraisemblablement pas d'accéder à une maîtrise de l'écrit adulte.

Par ailleurs, sauf à faire le pari que les autorités japonaises réussiront à faire en sorte que ces immigrés restent célibataires, ou que, s'ils se marient, ils ne le feront qu'avec des japonais(es), ou bien encore que s'ils se marient avec d'autres immigrés(ées), ils attendront d'être rentrés dans leur pays pour avoir des enfants, voire, encore, que s'ils sont mariés dans leur pays ils ne chercheront pas à faire venir leur famille, les écoles japonaises vont devoir, dans le futur, faire face à l'arrivée plus ou moins massive d'écoliers n'ayant ni les mêmes capacités linguistiques ni le même bagage culturel que les écoliers japonais. Or, nombre de ces immigrés risquent, comme c'est le cas dans les grands pays d'immi- gration, de faire pour leurs enfants le pari de l'intégration par l'école, alors qu'eux-mêmes seront sur le plan scolaire incapables de les aider. Que pourra l'école japonaise pour ces enfants? Comment gérera-t-elle la présence de ces enfants élevés au Japon mais dont les familles n'auront pas ellesmêmes accès à l'écrit - sinon à la langue - de ce pays ? Sans doute, au premier abord, ce problème ne paraît-il guère différent de celui que pose la scolarisation des enfants immigrés dans l'ensemble des pays industrialisés qui ont choisi d'ouvrir leurs frontières à l'immigration. Toutefois, la spécificité du système d'écriture et de la méthode d'enseignement de la lecture au Japon, de même que les différentes caractéristiques de l'école japonaise que nous avons présentées plus haut nous paraissent considérablement compliquer la tâche des responsables japonais par rapport à celle - déjà complexe - de leurs homologues occidentaux.

Le ministère de l'Éducation japonais a publié en avril 2005 une enquête relative à la situation linguistique des élèves étrangers fréquentant les écoles japonaises Nihongo shidô ga hitsuyô na gaikokujin jidô seito no ukeire jôkyô ni kansuru chôsa (heisei 16 nendo), littéralement: "Enquête relative aux conditions d'accueil des enfants étrangers ayant besoin d'un enseignement [spécial] en langue japonaise 2004 " (Monbukagakushô, 2005). Cette enquête est la dixième du genre menée au Japon depuis 1990 . Ses principaux résultats sont les suivants:

- en septembre 2004, le nombre d'élèves étrangers "ayant besoin d'un enseignement [spécial] de japonais " dans les écoles élémentaires, les collèges et les lycées japonais était de 19678 (soit environ $0,13 \%$ de l'ensemble des 14880000 enfants scolarisés au Japon en 2004) : 13307 écoliers et 5097 collégiens (93,5\%), 1204 lycéens $(6,1 \%)$ et 64 enfants scolarisés dans les écoles pour handicapés ou autres $(0,38 \%)$;

- ces élèves étaient scolarisés dans 5346 écoles publiques différentes (sur un total de 41200 établissements scolaires) : 3097 école élémentaires, 1783 collèges, 308 lycées, 40 écoles pour handicapés et autres ;

- leur répartition dans les écoles était la suivante : 2606 écoles scolarisaient un seul enfant étranger, 973 en scolarisaient deux, 427 en scolarisaient trois, 298 en scolarisaient quatre, et 1042 en scolarisaient cinq ou plus ; 
- les collectivités locales dans lesquelles étaient scolarisés moins de cinq étrangers étaient au nombre de 564 (56,9\%), 114 comptaient entre cinq et dix enfants étrangers (11,5\%), 211 $(21,2 \%)$ en comptaient entre dix et cinquante, $59(5,9 \%)$ en comptaient entre cinquante et cent, et $44(4,4 \%)$ plus de cent ;

- $84 \%$ (16 529) «seulement 》 de l'ensemble de ces enfants recevaient un enseignement spécial en japonais : 14460 écoliers et 4117 collégiens, 906 lycéens et 46 enfants scolarisés dans les écoles pour handicapés ou autres ;

- le séjour de ces enfants dans les écoles japonaises était de durée variable : $4390(22,3 \%)$ étaient scolarisés pour moins de six mois, 2585 entre six et douze mois $(13,1 \%), 4024$ entre un et deux ans $(20,4 \%), 2899$ entre deux et trois ans $(14,7 \%), 3022$ entre trois et cinq ans $(15,4 \%), 2496$ entre cinq et dix ans $(12,7 \%)$, 262 plus de dix ans $(1,3 \%)$;

- la langue maternelle de ces enfants était pour $35,7 \%$ d'entre eux le portugais, pour $23,5 \%$ le chinois, pour $14,9 \%$ l'espagnol, et les $25 \%$ restant avaient une autre langue maternelle: le tagalog $(9,1 \%)$, le coréen $(4,6 \%)$, le vietnamien $(3,6 \%)$, l'anglais $(2,5 \%)$, autres $(6,0 \%)$.

\section{Quels enseignements peut-on tirer} de ces chiffres?

La première constatation qu'il convient de faire est tout d'abord que le nombre d'élèves étrangers "ayant besoin d'un enseignement de japonais" continue de progresser d'année en année : évalué à 5463 en 1991, il était de 11553 en 1995 (Vaipae, 2000, p. 185) et donc de 19678 en 2004. Toutefois, dans le même temps, ce nombre semble s'être stabilisé ces six dernières années aux alentours de 19000 ; et, surtout, rapporté au nombre total d'élèves scolarisés, soit 0,13\% en 2004, il laisse également penser que le problème posé par les enfants étrangers dans les écoles japonaises reste encore aujourd'hui un épiphénomène: très peu d'écoles, très peu d'enseignants, très peu de collectivités locales sont concernés. La scolarisation de ces enfants est par ailleurs très courte dans le temps : elle dure moins de deux ans pour 55,8\% d'entre eux.

II convient ensuite de se demander ce que ces chiffres représentent véritablement. Dans les documents que nous avons consultés, la catégorie " enfant ayant besoin d'un enseignement [spécial] de japonais » n'est jamais clairement définie, de même que ne sont d'ailleurs jamais mentionnés les critères qui président à cette catégorisation, ni le nombre d'enfants étrangers " n'ayant pas besoin " d'un enseignement particulier. Les statistiques du ministère de la Justice japonais (Hômushô, 2003) relatives aux populations immigrées résidant dans l'archipel évaluent de leur côté à respectivement 127198 et 140413 le nombre des enfants étrangers âgés de zéro à neuf ans et de dix à dix-neuf ans vivant au Japon, soit 276611 enfants en tout. Même si ce dernier chiffre inclut les enfants d'âge pré-scolaire (zéro à cinq ans), l'écart qui existe entre ces 276611 enfants et les 19678 écoliers, collégiens et lycéens " ayant besoin d'un enseignement de japonais " évoqués dans l'enquête de 2004 étonne. II est vrai qu'il est difficile au vu de ces seuls chiffres de distinguer, par exemple, les enfants étrangers nés au Japon - éventuellement de parents étrangers euxmêmes nés au Japon - et les enfants étrangers nés hors du Japon dont les situations respectives recouvrent des besoins linguistiques différents. II y a toutefois de grandes chances que le nombre global des enfants étrangers ayant besoin d'un enseignement spécial de japonais soit en réalité, comme le signalent de nombreux observateurs, largement supérieur à celui des enfants identifiés par cette enquête (Vaipae, 2000 , p. 185). Fondé sur le seul signalement (forcément subjectif) des enseignants, le décompte du ministère manque en effet de précision, les enseignants surestimant souvent les capacités linguistiques de ces élèves - sans compter que nombre d'entre eux vont par ailleurs être tout simplement très réticents à signaler ces enfants comme "différents": "étant donné que l'expression "enfants ayant besoin d'un enseignement de japonais ", n'est pas clairement définie [...] les élèves qui ont les capacités de s'exprimer dans les conversations de tous les jours, ne sont pas considérés comme " ayant besoin d'un enseignement de japonais » "(Vaipae, 2000, p. 185).

Le deuxième sentiment que l'on a à la lecture de cette enquête est que le problème des enfants étrangers dans les écoles japonaises n'est pas encore clairement identifié ni mesuré par les autorités.

On voit bien, par ailleurs, au travers des chiffres relatifs à la durée de la scolarisation des élèves étrangers au Japon, que se pose la question du niveau linguistique acquis par ceux-ci au regard du "facteur temps": si, en 2004, 44,2 \% des enfants ont ainsi été scolarisés plus de deux ans dans le système éducatif japonais, seuls 1,3\% l'ont été plus de dix ans. Théoriquement seuls ceux-ci ont donc eu (ou auront) la possibilité de réellement parvenir à une maîtrise correcte de l'écrit. 
L'enquête ne dit rien toutefois des capacités des élèves étrangers ni - autre critère capital pour juger de l'accès à la maîtrise de l'écrit japonais - de leur âge d'entrée dans le système éducatif japonais. L'expérience des autres pays industrialisés montre en effet que non seulement beaucoup de temps est nécessaire aux enfants d'immigrés pour rattraper leur retard linguistique lorsqu'ils arrivent dans leur pays d'accueil, mais que, par ailleurs, plus ils y arrivent âgés plus cela devient difficile, voire impossible (Vaipae, 2000, p. 190-191). Mais de tout cela non plus, l'enquête de 2004, comme les précédentes, ne parle pas.

Cette enquête en revanche montre bien que, sur l'ensemble des collectivités locales japonaises, seuls dix départements (sur quarante-sept) et quatre-vingt treize des municipalités scolarisant des élèves étrangers (sur 992 concernées) avaient recruté des enseignants titulaires spécialisés dans l'enseignement du japonais; que seuls vingt départements et quatrevingt six municipalités organisaient des stages pour les enseignants non spécialisés; que seuls cinq départements et trente-cinq municipalités proposaient des matériaux pédagogiques spéciaux destinés aux élèves étrangers ; et que seuls cinq départements et cent vingt-cinq municipalités avaient mis au point des guides destinés à informer les parents de ces enfants sur les conditions de leur scolarité.

Ainsi, le ministère de l'Éducation japonais a beau affirmer que, non concernés par l'obligation scolaire, les enfants étrangers ont tout de même le droit, si leurs parents le souhaitent, d'être scolarisés dans une école élémentaire ou un collège publics japonais, et il a beau spécifier qu'ils doivent alors être traités de la même manière que les enfants japonais, notamment au regard de la gratuité de la scolarité et des manuels scolaires, dans la réalité toutefois, les chiffres de l'enquête le montrent sans ambiguïté, l'école japonaise n'est pas du tout prête à les accueillir.

L'affirmation de Vaipae (2000, p. 199) selon laquelle "les initiatives du ministère de l'Éducation vis-à-vis des enfants appartenant à une minorité linguistique relèvent du seul volontariat " reste d'actualité, et il suffit de faire quelques recherches sur Internet pour se rendre compte que la plupart des initiatives en la matière sont extérieures à l'école publique : associations, ONG, municipalités, etc. Les initiatives de ces dernières comme celles des collectivités locales en général tendent ainsi à " externaliser » le problème, à le « sortir » de l'école.
Tous les enfants étrangers qui arrivent ou arriveront au Japon dans le futur n'auront pas tous le même âge ni le même parcours scolaire - s'ils en ont un! Or l'âge d'arrivée constitue un point critique au regard de la réussite scolaire, l'âge de quinze/seize ans étant considéré comme un palier important dans les autres pays industrialisés (Maréchal, 2002). Mais qu'en serat-il au Japon? II y a de fortes chances que, pour toutes les raisons linguistiques et pédagogiques que nous avons exposées plus haut, cet âge critique en deçà duquel tous les efforts sont à l'avance voués à l'échec doive y être placé encore plus bas. Par ailleurs, les structures généralement mises en place dans les pays occidentaux (France, 2001) visent - dans l'idéal - à scolariser temporairement les enfants dans des classes spécifiques (ou dans des dispositifs spécifiques proposés parallèlement à leur inscription dans des classes "normales") afin de permettre à ces derniers d'intégrer le plus tôt possible - en situation de réussite - une classe du cursus ordinaire. Aussi nous faut-il reposer une nouvelle fois la question : comment mettre en place des dispositifs équivalents dans le système éducatif japonais avec tous les obstacles que nous avons évoqués, un système où domine par ailleurs une pédagogie livresque qui fait de la maîtrise de la lecture la carte d'accès à toutes les autres disciplines?

Pour les autorités japonaises, les options sont, de fait, des plus limitées et se résument à l'alternative suivante : soit réformer l'école pour faciliter l'accueil des élèves étrangers, soit scolariser ces derniers en dehors de celle-ci. On peut donc voir dans l'action des diverses organisations ainsi que dans les initiatives individuelles ou collectives que nous venons d'évoquer le souci et la volonté de contrebalancer les insuffisances de l'école japonaise en ce qui concerne l'accueil des enfants étrangers, mais on peut également discerner dans ce type d'interventions le début d'une politique qui pourrait se généraliser dans le futur: celle consistant à scolariser ces enfants en dehors du système éducatif japonais ou à les maintenir à l'intérieur du système éducatif public mais en les marginalisant au travers de parcours spécifiques - ce qui, du point de vue de la marginalisation, reviendrait au même.

\section{MULTILINGUISME ET CITOYENNETÉ}

L'enjeu ici n'est pas simplement scolaire. La présence de plus en plus nombreuse et visible des nouveaux arrivants va poser à terme non seulement la 
question de l'identité japonaise (qui est japonais ?), mais également celle de la citoyenneté au Japon (qui participe activement à la vie de la cité et s'y épanouit ?). Et on peut imaginer que fort probablement l'un des critères qui présidera à la définition de cette nouvelle citoyenneté sera la maîtrise de la langue. Comme l'écrit Philippe Pelletier : "L'arrivé des immigrants asiatiques nyûkâmazu [...] dont la problématique de cohabitation ou d'intégration est très différente de celle des ôrudo-kâmazu (old comers), chamboule les conceptions culturalo-ethnicistes de l'identité japonaise, en particulier à travers le cas des nikkeijin qui apparaissent comme un nouveau type de japonais non-japonais. » (Pelletier, 2003, p. 201)

« De plus en plus d'Occidentaux arrivent à parler un japonais très correct alors que les nikkeijin le parlent mal, bien que "typés japonais ». Cette situation apparaît comme "non naturelle » pour les japonais [...] L'existence d'étrangers finalement « très japonais " et de japonais " ethniquement japonais " (les nikkeijin) mais qui, dans la langue et le comportement, ne sont pas de "vrais japonais ", perturbe l'ancienne adéquation entre identité ethnique et identité socio-culturelle. Or la vulgate nippone posait cette adéquation comme la quintessence et la particularité de la japonité. La croyance affirmant que ceux qui parlent japonais et qui ont une apparence physique japonaise ne peuvent être que des Japonais est ainsi remise en cause par l'expérience d'une « dissonance des limites » (Ibid., p. 170).

Le point de vue qui lie la question de l'identité japonaise à celle de la maîtrise de la langue japonaise nous paraît particulièrement pertinent. On peut cependant sans doute aller un peu plus loin si on considère non plus seulement la capacité à parler et à comprendre le japonais, mais également celle de le lire et de l'écrire. En effet, comme le souligne Unger (1987, p. 104): "L'attachement des japonais aux kanji est étroitement lié à l'expérience partagée de maîtriser un corps complexe de savoir qui définit l'appartenance au groupe ».

Est-ce à dire que sont ou seront considérés comme japonais ceux (et seulement ceux) qui, en plus de le parler, lisent et écrivent le japonais ? Il est difficile d'aller jusque-là aujourd'hui, même si le discours sur l'illettrisme qui est tenu au Japon depuis les années 1950 laisse entrevoir cette possibilité. En effet, reproduire, comme le font régulièrement les responsables japonais et certains spécialistes occidentaux, enonobstant l'absence de toute étude nationale fondée sur des critères scientifiques objectifs (voir Galan, 2006), le discours selon lequel il n'y aurait au Japon que 0,1 ou $0,3 \%$ d'illettrés, c'est accepter de ne considérer la population japonaise qu'une fois débarrassée de tout ce qui ne peut être régardé comme étant purement japonais : burakumin (5), Coréens, handicapés physiques et mentaux, returnees de Chine, travailleurs journaliers ayant perdu toute hygiène de vie propre à perpétuer la maîtrise de la lecture, réfugiés politiques et travailleurs immigrés en règle ou clandestin, etc. - sans oublier les enfants déscolarisés. Car sous le discours officiel sur l'illettrisme se cache un autre discours que l'on peut résumer ainsi : tous les "vrais" japonais, tous les japonais "normaux ", savent lire. II suffit de renverser les deux termes de ce raisonnement pour établir que tous ceux qui ne savent pas lire ne sont pas japonais et ne peuvent le devenir.

Dans le même ordre d'idée, on peut également poser que l'accès à l'écrit définit ou définira un niveau supérieur de citoyenneté au Japon. Dans tout pays, il existe en effet un lien fondamental entre la citoyenneté et la maîtrise de la langue, le sentiment d'appartenir à une société passant en grande partie par la certitude d'avoir accès à l'intégralité des informations qu'elle rend disponibles. Apprendre non seulement la langue, mais également à lire et à écrire dans cette langue, c'est entrer dans un processus qui rend possible l'intégration sociale dans le pays d'accueil ou, du moins, pour reprendre les termes du rapport Japan's Goals in the Twenty-First Century, s'agit-il là d'une des conditions sine qua non qui permet aux "étrangers de vivre normalement et confortablement » (Shushô kantei 2000, ch. 1).

Le concept de global literacy qui figure également dans ce rapport fait bien sûr référence à la nécessité pour la population japonaise, si elle veut accéder à une nouvelle citoyenneté "mondiale " au cours du $\mathrm{xxl}^{\mathrm{e}}$ siècle, de parvenir à une maîtrise réelle de l'anglais (et de l'informatique). On peut cependant voir également, en filigrane, dans le concept d'" accès à l'information " qui est ici mis en avant comme la clef de cette citoyenneté mondiale, une condition essentielle de l'exercice d'une citoyenneté "locale " pour les populations non japonaises vivant sur l'archipel. Mais quel sera alors pour ces populations la façon d'accéder librement à l'information et au savoir? Sera-ce via une langue anglaise devenant seconde langue nationale, comme cela fut récemment envisagé ? Sera-ce via la seule langue japonaise? Sauf à imaginer un Japon du futur optant pour une solution à la canadienne, on peut avancer que ce sera vraisemblablement, et pendant longtemps encore, comme aujourd'hui, via la seule langue japonaise... 
Or si l'enseignement actuel du japonais aux enfants des «minorités linguistiques", comme on les appelle également, " peut contribuer à augmenter leurs capacités de communication sociale, il est loin de leur permettre d'acquérir les connaissances nécessaires à la construction des capacités linguistiques académiques »(Vaipae, 2000, p. 234) - et, ajouterions-nous, bien plus loin encore de donner à quiconque les moyens d'exercer une quelconque citoyenneté, s'il est vrai que celle-ci exige un accès minimum à l'information disponible dans la langue écrite du pays.

Une nouvelle distribution des populations pourrait ainsi voir progressivement le jour qui opposerait deux catégories d'individus - et donc deux niveaux de citoyenneté -, ceux qui auront accès à l'écrit et ceux qui n'y auront pas accès. Le critère du temps passé au Japon s'ajoutant à celui du temps passé à étudier la langue japonaise conditionnera alors l'appartenance des individus à l'une ou l'autre de ces catégories.

\section{CONCLUSION}

Pluralisme linguistique, intégration par la langue, internationalisation, adoption de l'anglais comme langue nationale..., quelle que soit la direction dans laquelle la situation linguistique du Japon évoluera dans le futur ou quelle que soit la direction dans laquelle souhaiteront la faire évoluer les dirigeants de ce pays, ces derniers ne pourront vraisemblablement pas faire l'économie d'une nouvelle réflexion sur le système d'écriture japonais. Nous partageons ainsi pleinement l'avis d'Unger lorsqu'il écrit: « La société japonaise peut bien avoir tourné le dos pour le moment aux réformes de son système d'écriture, les problèmes sous-jacents n'en ont pas disparu pour autant » (Unger, 1996, p. 125).

En effet, si l'on considère que les Japonais ont besoin de neuf (ou douze) ans pour accéder pleinement à l'écrit adulte, on peut poser comme principe que toute personne - et notamment les étrangers et les enfants d'étrangers vivant au Japon - qui n'aura pas bénéficié de ces neuf (ou douze) années de scolarité au Japon ne pourra espérer parvenir à la maîtrise de l'écrit. Bien sûr la maturité (pour les élèves les plus âgés ou les adultes) et la motivation peuvent jouer et réduire la durée de cet apprentissage, mais au bout du compte pas autant sans doute qu'on pourrait l'espérer du fait des limites (actuelles ?) de la mémoire humaine.
Que le Japon choisisse de devenir une société ouverte, plurilingue et/ou multiculturelle, qu'il opte pour une politique d'intégration de ses travailleurs immigrés, ou qu'il mêle les deux options, se posera alors aussitôt la question de l'accès à l'écrit pour tous les individus vivant au Japon, avec en arrièreplan la question de la citoyenneté et celle de l'égalité. L'école japonaise se retrouvera alors quant à elle face à un véritable défi : celui de devoir faire accéder à l'écrit un grand nombre d'individus dont la langue maternelle et/ou celle parlée à la maison ne sont pas le japonais.

Si le Japon opte en revanche pour une politique de développement séparé, nul doute alors que son école et son système d'écriture actuels constitueront deux des meilleurs outils de cette politique, et les remparts les plus efficaces à toute altérité de la « japonité » telle que celle-ci est actuellement définie. Mais la question reste cependant de savoir si cette position sera " viable » sur le long terme, ou si elle est en elle-même raisonnable.

En effet, s'il est encore trop tôt pour affirmer que c'est l'accès à l'écrit qui à l'avenir définira l'identité japonaise ou jouera un rôle majeur dans sa définition (encore que le discours actuel sur l'illettrisme ne fasse pas autre chose), il paraît évident en revanche que cet accès conditionnera de plus en plus dans le futur l'exercice de la citoyenneté japonaise.

Sur un plan plus pédagogique - mais restant lié au point précédent - l'enseignement de la langue aux apprenants non japonais devra à l'évidence lui aussi faire l'objet d'une réflexion approfondie dans laquelle seront pris en compte non seulement les individus qui ont un fort désir d'apprendre la langue japonaise, mais également ceux - enfants ou adultes - qui n'en ont ni l'envie ni la motivation. Dans le même ordre d'idée, il sera intéressant de voir si dans le futur ce débat continuera de relever presque exclusivement des ONG et des collectivités locales, ou encore d'organismes tels que la Fondation du Japon, ou si l'école - et ses enseignants s'y impliqueront également.

À terme, se posera sans doute d'ailleurs la question du rôle et de la finalité de l'école japonaise. Une redéfinition de ce rôle et de cette finalité ne manquera probablement pas de s'opérer au travers notamment du concept de « différence » - une différence acceptée, vécue comme une réalité voire une richesse, et non plus seulement comme synonyme d'exclusion. En ce sens, on peut penser qu'une réflexion bien menée sur les problèmes que pose la 
scolarisation des enfants étrangers pourrait profiter à l'ensemble des enfants japonais: elle ferait notamment prendre conscience aux parents et aux éducateurs japonais qu'il est normal que les enfants n'avancent pas tous au même rythme, que leurs besoins et leurs capacités divergent d'un individu à l'autre, ou encore, en ce qui concerne plus particu- lièrement la lecture, que tous les enfants ne se l'approprient pas de la même façon.

\section{Christian Galan}

cgalan@univ-tlse2.fr

Université Toulouse-Le Mirail

CNRS-Centre de recherche sur le Japon

\section{NOTES}

(1) Nous avons longuement décrit cette méthode dans plusieurs de nos précédents travaux et nous ne présentons ici que les grandes lignes de celles-ci (pour plus de détails, voir notamment Galan 1995a \& 2001).

(2) Pour une présentation des écoles préélémentaires japonaises, voir Galan, 1995b.

(3) Les juku sont des écoles qui sont en dehors du système éducatif et dans lesquelles les enfants se rendent pour consolider ce qu'ils étudient à l'école publique ou pour prendre de l'avance sur les programmes. Ces écoles sont privées, payantes et leurs taux de fréquentation augmentent proportionnellement au niveau d'étude des enfants.
(4) Nous préférons cette formulation plutôt que de parler d'homogénéité linguistique, formulation qui nous permet de ne pas faire un cas particulier des enfants "étrangers " appartenant aux différentes communautés vivant au Japon depuis plusieurs générations.

(5) Les burakumin constituent une communauté de plus de deux millions de personnes vivant dans des ghettos. Bien que Japonais " de souche ", ils continuent d'être victimes d'une discrimination liée, au regard du shintô, à l'impureté des métiers de leurs ancêtres qui amenaient ces derniers à côtoyer les cadavres et le sang.

\section{BIBLIOGRAPHIE}

Enel F. \& Delesalle C. (2001). « Les plates-formes d'accueil des primo-arrivants: l'enquête auprès des bénéficiaires ", Migrations études, $\mathrm{n}^{\circ} 103$ bis, $\mathrm{p}$..

France: Ministère de L'Éducation nationale (2001). "Les élèves nouveaux arrivants non francophones et leur scolarisation dans les différents dispositifs d'accueil ». Note d'information, $\mathrm{n}^{\circ}$ 01-57.

GALAN C. (1995). «Lecture et illettrisme : l'exception japonaise, discours et réalité ». In Japon pluriel. Arles: P. Picquier, p. 143-150.

GALAN C. (1995). " L'importance de l'« environnement » dans l'apprentissage de la lecture au Japon », Psychologie \& éducation, $\mathrm{n}^{\circ} 20$, p. 49-69.

GALAN C. (2001). L'enseignement de la lecture au Japon : politique et éducation. Toulouse: Presses universitaires du Mirail.

GALAN C. (2006). « Le (s) discours sur l'illettrisme dans le Japon contemporain ». In J. Fijalkow \& C. Galan (dir.), Langue et lecture au Japon. Arles : P. Picquier [à paraître].

HômuSHô (2003). Heisei 15 nenmatsu genzai ni okeru gaikokujin tôrokusha tôkei ni tsuite. Document au format PDF, disponible sur Internet à l'adresse : http://www. moj.go.jp/PRESS/040611-1/040611-1-1.pdf [consulté le 28 novembre 2005].

MAKITA K. \& SAKAMOTO T. (1973). “ Japan ». In J. Downing, Comparative Reading (Cross-National Studies of Behavior and Processes in Reading and writing). New York \& Toronto: Macmillan, p. 440-465.

MARÉCHAL C. (2002). "Société, éducation et diversité culturelle ». Document disponible sur Internet à l'adresse : http://www.ac-rennes.fr/pedagogie/hist_geo/ResPeda /Migrations/catherine/Copie\%20de \%20SOCIETE\% 20EDUCATION\%20ET\%20DIVERSITE\%20CULTUREL LE2.htm [consulté le 28 novembre 2005].
MONBUKAGAKUSHÔ (2005). Nihongo shidô ga hitsuyô na gaikokujin jidô seito no ukeire jôkyôtô ni kan suru chôsa (Heisei 16 nendo) no kekka. Document au format PDF disponible sur Internet à l'adresse : http://www.mext.go. jp/b_menu/houdou/17/04/05042001/010.pdf [consulté le 28 novembre 2005].

Pelletier P. (2003). Japon: crise d'une autre modernité. Paris : Belin : La Documentation française.

SHUSHÔ KANTEI (2000). 21 seiki nihon no kôsô: Nihon no furontia wa nihon no naka ni aru: jiritsu to kyôji de kizuku shinseiki. Disponible sur Internet à l'adresse: http://www.kantei.go.jp/jp/21century/houkokusyo/ index1.html [consulté le 28 novembre 2005].

SHUSHÔ KANTEI (2000). Japan's Goals in the Twenty-First Century: The Frontier Within : Individual Empowerment and Better Governance in the New Millennium, Disponible sur Internet à l'adresse : http://www.kantei.go.jp/jp/21 century/report/htmls/index.html [consulté le 28 novembre 2005].

SÔMUSHÔ - TÔKEIKYOKU (2000). Future Population Projection (2005-2100). Document au format PDF disponible sur Internet à l'adresse : http://www.stat.go.jp/english/data/ nenkan/y0202000.pdf [consulté le 28 novembre 2005].

UNGER J. M. (1987). The Fifth Generation Fallacy: Why Japan Is Betting Its Future on Artificial Intelligence. New York: Oxford University Press.

UNGER J. M. (1996). Literacy and Script Reform in Occupation Japan. New York : Oxford University Press.

VAIPAE S. (2000). «Language Minority Students in Japanese Public Schools ». In M. G. Noguchi \& S. Fotos [éd.] (2000). Studies in Japanese Bilingualism. Clevendon: Multilingual Matters, p. 184-233. 